\title{
Contact forces between a particle and a wet wall at both quasi- static and dynamic state
}

\author{
Huang Zhang ${ }^{1}$, Sheng Chen ${ }^{1}$, and Shuiqing $\mathrm{Li}^{1, *}$ \\ ${ }^{1}$ Key Laboratory of the Thermal Science and Power Engineering of Ministry of Education, Department of Thermal Engineering, \\ Tsinghua University, Beijing 100084, China
}

\begin{abstract}
The contact regime of particle-wall is investigated by the atomic force microscope (AFM) and theoretical models. First, AFM is used to measure the cohesive force between a micron-sized grain and a glass plate at quasi-static state under various humidity. It is found out that the cohesive force starts to grow slowly and suddenly increase rapidly beyond a critical Relative Humidity (RH). Second, mathematical models of contacting forces are presented to depict the dynamic process that a particle impacts on a wet wall. Then the energy loss of a falling grain is calculated in comparison with the models and the experimental data from the previous references. The simulation results show that the force models presented here are adaptive for both low and high viscosity fluid films with different thickness.
\end{abstract}

\section{Introduction}

Wet granular materials are most common in our daily environment as well as in many engineering processes $[1,2]$. The packing or flow behaviour of wet granular media is closely relevant with the interactions at the grain level [1]. Interstitial liquid between the wet grains induces the granular matter cohesive arising from the capillary forces [3]. The experimental measurements and theoretical derivation to determine the magnitude of capillary force between Geldart-D coarse particles have been well established at quasi-static state [3-5]. For micron-sized grains, other adhesion effects caused by van der Waals force, electrostatic force, etc., become significant and complex [6]. So it is interesting to investigate how capillary force behaves in such a small length-scale. It seems that AFM offers a 'prototypical' approach to measure the contact forces in these conditions [7].

When the wet granular media is agitated, partially wetted grains are impacting and separating with each other [8]. The thin liquid layer covering a wet grain helps to push or pull the other grain [8]. Due to the hydrodynamic and viscous fluid flows in this liquid film, the first-contact energy loss [9] of wet particles is higher than that of dry ones [10]. Experiments can determine the particle velocities before and after collisions, so that the first-contact energy loss is obtained according to the velocity differences [10]. However, the details of contact mechanism of wet particles are relied on the theoretical analysis [11].

The structure of this paper is organized as following. First, the capillary force between a micron-sized grain and a plate (the plate can be considered as a sphere with zero curvature) is measured by AFM under different humidity. Second, the force models of a grain colliding with a wet wall are established in order to analyze the contacting mechanism, especially the first-contact energy loss between the grain and the wet wall.

\section{Experiment setup}

Fig. 1 shows the humidity control system and AFM (CSPM5500) setup. The circuit of nitrogen flows going through the water tank is to entrain liquid into the AFM cell, while another circuit of dry nitrogen is to control the magnitude of RH. The RH in AFM cell is adjusted to range from $2 \%$ to $85 \%$. RH is measured by VAISALA sensor. $\mathrm{A} \mathrm{SiO}_{2}$ particle with diameter $15.52 \mu \mathrm{m}$ is glued to the original AFM tip to form a spherical tip.

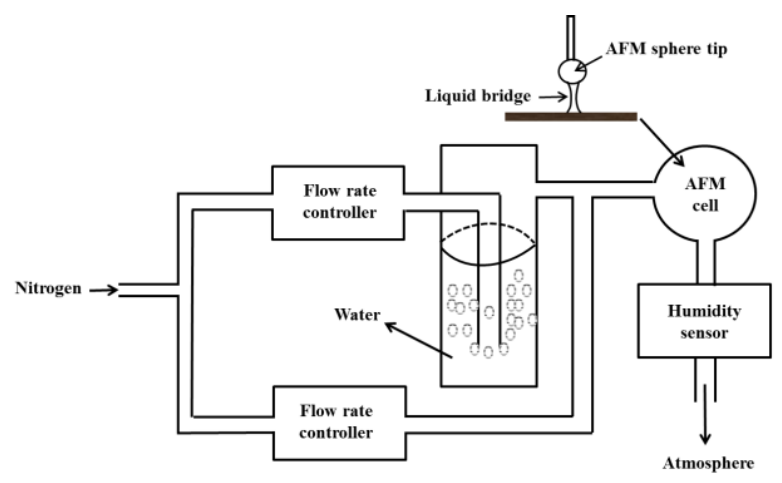

Fig. 1. Humidity control system and AFM setup.

\section{Theoretical models}

This part focuses on the establishing of the dynamic model that a grain impacts on a wet wall. Fig. 2 performs

Corresponding author: lishuiqing@,tsinghua.edu.cn 
the penetration and rebound schematic diagram. In this figure, $\beta$ is the angle between the three-phase contact line and the $y$ axis, and $\theta$ is the contact angle of solid and liquid. When the falling particle contacts the liquid layer (stage I), the fluids are draining out in the thin gap. During this process, the particle undergoes the resistance from the surface integration of the fluid stress at the interface between the particle and the liquid layer [12]. After penetrating the liquid film, the grain is colliding with the wall (stage II), and then, the grain rebounds through the fluid layer (stage III). After that, the liquid bridge pulls the particle until it is ruptured.
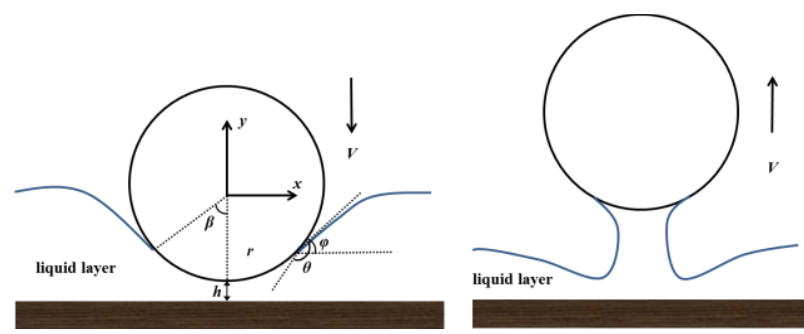

Fig. 2. Schematic diagram of a particle penetrating and separating with a liquid layer.

In stage I, the particle undertakes gravity force, surface tension force and the fluid force resulting from the fluid flows in the thin layer. This fluid force consists of three parts: unsteady, buoyance and resistance term $[11,12]$. When the particle Reynolds number $\operatorname{Re}_{p}$ is sufficiently small, the buoyance and resistance terms are converted into the lubrication force $[3,11]$. The motion equation of the sphere at this stage is shown as below

$$
m_{p} \frac{d \boldsymbol{v}}{d t}=\boldsymbol{F}_{g}+\boldsymbol{F}_{s}+\boldsymbol{F}_{a}+\boldsymbol{F}_{b}+\boldsymbol{F}_{d}
$$

where $m_{p}, \boldsymbol{v}, \boldsymbol{F}_{g}, \boldsymbol{F}_{s}, \boldsymbol{F}_{a}, \boldsymbol{F}_{b}$ and $\boldsymbol{F}_{d}$ are particle mass, particle velocity, gravity force, surface tension force, unsteady force, buoyance force and drag force, respectively. These forces are expressed as: $\boldsymbol{F}_{g}=(4 / 3)$ $\pi r^{3} \boldsymbol{g}, \quad \boldsymbol{F}_{s}=-2 \pi \gamma r \sin \beta \sin \varphi \boldsymbol{v} /|\boldsymbol{v}|, \quad \boldsymbol{F}_{a}=-(1 / 6) \pi r^{3} \rho_{f}\left(\cos ^{3} \beta-\right.$ $3 \cos \beta+2) \mathrm{d} \boldsymbol{v} / \mathrm{d} t, \boldsymbol{F}_{b}=-(1 / 3) \pi r^{3} \rho_{f}(2+\cos \beta)(1-\cos \beta)^{2} \boldsymbol{g}, \boldsymbol{F}_{d}=$ $-(1 / 2) \pi C_{d} \rho_{f}(r \sin \beta)^{2}|\boldsymbol{v}| \boldsymbol{v}$, where $r, \boldsymbol{g}, \gamma, \rho_{f}$, and $C_{d}$ are particle radius, gravity acceleration, surface tension coefficient, liquid density, and drag coefficient, respectively $[11,12] . C_{d}$ is related with $\mathrm{Re}_{p}$, which are defined as below [11]

$$
C_{d}=\frac{24}{\operatorname{Re}_{p}}+\frac{4}{\sqrt{\operatorname{Re}_{p}}}+0.4, \operatorname{Re}_{p}=\frac{\rho_{f}|v| d}{\mu},
$$

where $d, \mu$ are particle diameter and liquid viscosity. Particularly, if the flow Reynolds number $\mathrm{Re}_{f}$ in the liquid film is small, buoyance force and drag force are eventually lubrication force $[3,13] . \quad \mathrm{Re}_{f}$, which is estimated by considering mass conservation in the liquid layer, is performed as $\mathrm{Re}_{f}=\rho_{f}|\boldsymbol{v}|(r h)^{0.5} /(2 \mu)$, where $h$ is the gap distance between the bottom of sphere and the wall [3]. Here, we assume that if $\operatorname{Re}_{f}$ is less than one, $\boldsymbol{F}_{b}$ and $\boldsymbol{F}_{d}$ become to be the lubrication force $\boldsymbol{F}_{l}$, which is shown as $\boldsymbol{F}_{l}=-6 \pi \mu r^{2}|\boldsymbol{v}| /\left(h+h_{r}\right)$ [11]. Here $h_{r}$ is the surface roughness, which is used to avoid the physical singularity when $h$ is approaching zero [14].

In stage II, the grain collides with the wall with no interstitial fluid evolves any more [10]. We use $\boldsymbol{v}_{1}$ and $\boldsymbol{v}_{2}$ to represent the particle velocities that are just contacting and leaving the wall surface. Therefore, $\boldsymbol{v}_{1}$ and $\boldsymbol{v}_{2}$ are correlated with the restitution coefficient between the particle and the dry wall $[6,10]$, which is given as $\boldsymbol{v}_{2}=$ $e_{\text {dry }} \boldsymbol{v}_{1}$, where $e_{\text {dry }}$ is the restitution coefficient for dry condition.

In stage III, the grain starts to leave the wet wall. The forces that the particle undergoes in this period are nearly the same as those in stage I. While, $\boldsymbol{F}_{s}$ is replaced by the liquid bridge force $\boldsymbol{F}_{c}$ [4], which is given as below

$$
\boldsymbol{F}_{c}=-2 \pi \gamma r \cos \theta\left(1-\frac{1}{\sqrt{1+\frac{2 V_{b}}{\pi r\left(h+h_{r}\right)^{2}}}}\right) \frac{\boldsymbol{v}}{|\boldsymbol{v}|} .
$$

$V_{b}$ is the liquid bridge volume, which is estimated as $d^{3} / 16$ [10]. The rupture distance $h_{\text {rup }}$ is crucial to determine the breaking point of the liquid bridge. The evolution of $h_{\text {rup }}$ is expressed as [5]

$$
h_{\text {rup }}=(1+\theta)(1+\sqrt{\mathrm{Ca}}) V_{b},
$$

where $\mathrm{Ca}$ is the capillary number that equals to $\mu|\boldsymbol{v}| / \gamma$. Otherwise, $\beta$ and $\theta$ keep constant where $\beta=120^{\circ}$ [16] and $\theta=111^{\circ}[15]$ in stage I, and, $\beta=80^{\circ}$ [16] and $\theta=$ $10^{0}$ [17] in stage III. Additionally, $\varphi$ equals $\beta+\theta-\pi$ [12].

\section{Results and discussions}

Fig. 3 gives the AFM measurement of the critical cohesive force $F_{\text {crit }}$ under different RH. In general, the magnitude of $F_{\text {crit }}$ grows with increasing RH. $F_{\text {crit }}$ rises slowly when RH is below the critical RH that is around $65 \%$. While, $F_{\text {crit }}$ rises up rapidly as RH is beyond $65 \%$. Actually, the surfaces of both $\mathrm{SiO}_{2}$ particle and glass plate are not smooth (the average roughness is about $2.98 \mathrm{~nm}$ by AFM measurement). As RH ranges from $3 \%$ $\sim 65 \%$, liquid could only exist within the surface concave due to capillary condensation [7]. Hence, the liquid connections can hardly contribute to the increment of the total cohesive force. When RH is high (beyond $65 \%$ in this case), still due to the capillary condensation, liquid could fill up the gap between the micron-sized particle and the plate. Then, the liquid volume $V_{b}$ in the gap rises with the growing $\mathrm{RH}$. Because the distance between the particle and the wall is constant, $F_{\text {crit }}$, which is now estimated by Eq. (3) $[4,7]$, would become larger and larger with increasing $V_{b}$.

The occurrence of critical RH needs further analysis. In equilibrium state, Kelvin equation describes the relationship between the shape of liquid concave and RH, which is shown as below,

$$
r_{c}=\frac{2 \gamma V_{m}}{R_{g} T \log (\mathrm{RH})},
$$


where $r_{c}, \gamma, V_{m}, R_{g}$, and $T$ are liquid concave radius, surface tension, liquid molar volume, ideal gas constant, and temperature. When the condensate liquid just fills up the surface roughness, $r_{c}$ is estimated as $-2.98 \mathrm{~nm}$, whose magnitude is supposed to be the average roughness. We take $T$ as $20^{\circ} \mathrm{C}$, so RH is calculated as $69.6 \%$ by Eq. (5), which is very close to the critical RH (65\%) from Fig. 3. Further, suppose the gap between the particle and the plate is filled with liquid if RH is approaching to $90 \%$ or higher. The contact force is regarded as $F_{\text {crit }} \sim \pi \gamma d$ [3]. According to the material properties, $F_{\text {crit }} \sim \pi \times 73 \times 10^{-}$ ${ }^{3} \times 15.52 \times 10^{-6} \mathrm{~N} \sim 3600 \mathrm{nN}$, which is more or less close to the maximum value of $F_{\text {crit }}$ (about $2000 \mathrm{nN}$ ) in Fig. 3 .

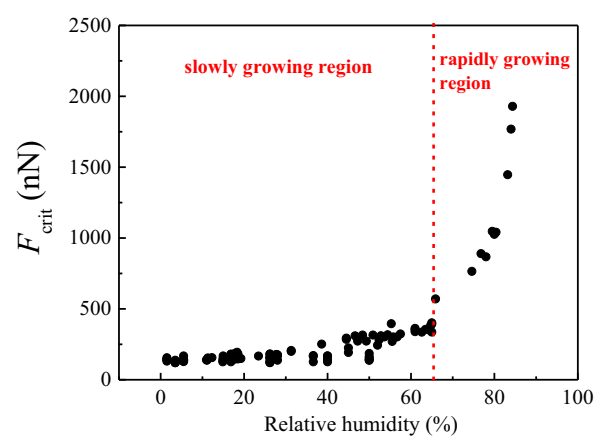

Fig. 3. Critical cohesive force $F_{\text {crit }}$ increases with relative humidity by AFM measurement.

Eq. (1) quantitatively capture the essential features of the impacting process in Fig. 2. In stage I, the total distance that the grain moves is the initial thickness of liquid layer $\delta$. Substituting the impacting velocity of particle $\boldsymbol{v}_{0}$ into Eq. (1), we can get $\boldsymbol{v}_{1}$ after numerically solving this Ordinary Differential Equation (ODE, and the numerical toolkit used here is ODE45 in MATLAB R2010a). Then, $v_{3}$ is also obtained from Eq. (1), in which $\boldsymbol{F}_{s}$ is instead of $\boldsymbol{F}_{c}$. Therefore, the first-contact energy loss is expressed as $0.5 m_{p}\left(\left|v_{0}\right|^{2}-\left|v_{3}\right|^{2}\right)$. In order to verify these theoretical models, $\Delta E_{\text {wet }}$ that indicates the energy loss during the process of the grain interacting with the liquid film, is compared with the experimental data in reference [10]. Obviously, $\Delta E_{\text {wet }}$ equals to $0.5 m_{p}\left(\left|v_{0}\right|^{2}-\right.$ $\left.\left|v_{3}\right|^{2}-\left|v_{2}\right|^{2}+\left|v_{1}\right|^{2}\right)$. Meanwhile, according to an energy budget analysis referred in ref. [10], $\Delta E_{\text {wet }}$ mainly consists of viscous dissipation and fluid material acceleration, which are given by [10]

$$
\begin{gathered}
\Delta E_{\mathrm{wet}}=\Delta E_{\mathrm{vis}}+\Delta E_{\mathrm{acc}}, \\
\Delta E_{\mathrm{vis}}=\frac{3}{2} \pi \mu d^{2}\left|\boldsymbol{v}_{0}\right|\left(\ln \frac{\delta}{h_{r}}+\ln \frac{h_{r u p}}{h_{r}}\right), \\
\Delta E_{\mathrm{acc}}=\frac{3 \rho_{f}}{2 \rho_{s}}\left(\frac{\delta}{d}-\frac{3}{5}\left(\frac{\delta}{d}\right)^{2}+\frac{2}{3}\left(\frac{\delta}{d}\right)^{3}\right) m_{p} v_{0}^{2} .
\end{gathered}
$$

Here $d_{\text {rup }}$ is considered as $2 d$ according to the observation in ref. [10]. The material properties of two kinds of fluid M5 and M50 are given in Table 1. The restitution coefficient $e_{\text {dry }}$, particle roughness $h_{r}$, particle density $\rho_{s}$ and diammeter $d$ are $0.985,5 \mu \mathrm{m}, 2580 \mathrm{~kg} / \mathrm{m}^{3}$ and $5.5 \mathrm{~mm}$, respectively [10].

Table 1. The material properties of M5 and M50 [10].

\begin{tabular}{|c|c|c|c|}
\hline Liquid & $\rho_{f}\left(\mathrm{~kg} / \mathrm{m}^{3}\right)$ & $\gamma(\mathrm{mN} / \mathrm{m})$ & $\mu(\mathrm{mPa} \cdot \mathrm{s})$ \\
\hline M5 & 925 & 19.2 & 4.6 \\
\hline M50 & 965 & 20.8 & 48 \\
\hline
\end{tabular}

As seen in Fig. 4(a), $\Delta E_{\text {wet }}$ predicted by our models is in good agreement with the experimental data. Generally, $\Delta E_{\text {wet }}$ increases with $\left|\boldsymbol{v}_{0}\right|$. Also, the more thick $\delta$ is, the larger $\Delta E_{\text {wet }}$ is dissipated. Remarkably, the energy loss of the particle impacting into the liquid M50 is much higher than that of the liquid M5. For instance, $\Delta E_{\text {wet }}$ is around $20 \mu \mathrm{J}$ as $\left|v_{0}\right|$ is $1 \mathrm{~m} / \mathrm{s}$ in the liquid M5 case, which is nearly one third of that in the same case of liquid M50.

Although the results obtained from Eqs. (6-8) qualitatively follow the tendency of $\Delta E_{\text {wet }}$ versus $\left|\boldsymbol{v}_{0}\right|$ and $\delta$, it deviate a lot in case of liquid M5. However, the accuracy of Eqs. (6-8) is highly promoted when calculating $\Delta E_{\text {wet }}$ in liquid M50. In contrast, $\Delta E_{\text {wet }}$ obtained by our model can still agree well with the experimental data of liquid M50, but they are not as well as the results by Eqs. (6-8) in this case. The viscosity of liquid M50 is nearly one thousand times larger than M5. Eq. (7) can be considered as the intergration of $\boldsymbol{F}_{l}$, which is given as below

$$
\Delta E_{\mathrm{vis}}=\int_{h_{r}}^{d_{r u p}}+\int_{\delta}^{h_{r}}\left(\boldsymbol{F}_{l} \cdot d \boldsymbol{x}\right)=\int_{h_{r}}^{d_{r u p}}+\int_{\delta}^{h_{r}}\left(\frac{3 \pi \mu d^{2} \boldsymbol{v}}{2 x} \cdot d \boldsymbol{x}\right) .
$$

Eq. (7) is obtained from integrating Eq. (9) by taking $v$ as $\boldsymbol{v}_{0}$, which should be larger than the particle velocity in actual situation. This is the reason that Eqs. (6-8) could get good results in the viscosity dominated case (M50), but fail in the less viscosity case (M5). However, as seen from Fig. 4, our model is adaptive for both low and high viscosity fluid. Furthermore, our model could predict the particle velocity at any given time, which is convenient to analysis the contacting mechanism of the particle with the liquid layer.

Fig. 4 indicates that $\Delta E_{\text {wet }}$ depends on $\boldsymbol{v}_{0}, \mu$, and $\delta$, etc. A usually used dimensionless numer to clarify the energy loss process is Stokes numer St, which is defined as $\rho_{s} d \mid \boldsymbol{v}_{0} / /(9 \mu)[3,10-11,13]$. In the main diagram of Fig. 5, the experimental points of $\Delta E_{\text {wet }} / E_{0}$ ( $E_{0}$ is the initial kinetic energy of an impact particle) are almost collapsed into a fitting curve, whose form is as $a \mathrm{St}^{-2}+b \mathrm{St}^{-1}+c$. Similarly, the scatter results by our model are also fitted by a curve with the same form as in the main diagram.

As seen in Fig.5, the ratio of $\Delta E_{\text {wet }}$ to $E_{0}$ does not exceed 0.4 when $\mathrm{St}^{-1}$ ranges from 0 to 0.005 . With increasing $\mathrm{St}^{-1}$, this ratio grows up to around 0.8 . The law of the fitting curve is not occasional, which is intrinsically shown as following: $\Delta E_{\text {wet }} / E_{0}$ is direct propotion to $e_{n}^{2}$ [10], where $e_{n}$ is the coefficient of 
restitution. Further, $e_{n} \sim\left(1-\mathrm{St}^{-1}\right)[3]$ to lead that the curve of $\Delta E_{\text {wet }} / E_{0}$ is in the form of $a \mathrm{St}^{-2}+b \mathrm{St}^{-1}+c$.
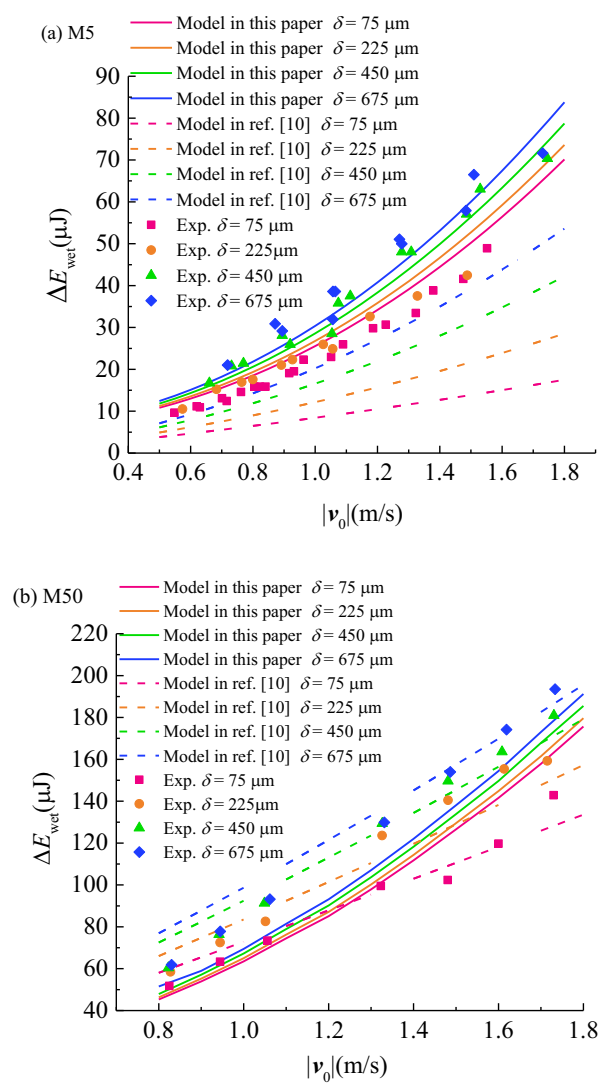

Fig. 4. $\Delta E_{\mathrm{wet}}$ caclulated by the model in this paper is compared with both the model as shown in Eqs. (6-8) and experimental data in reference [10].

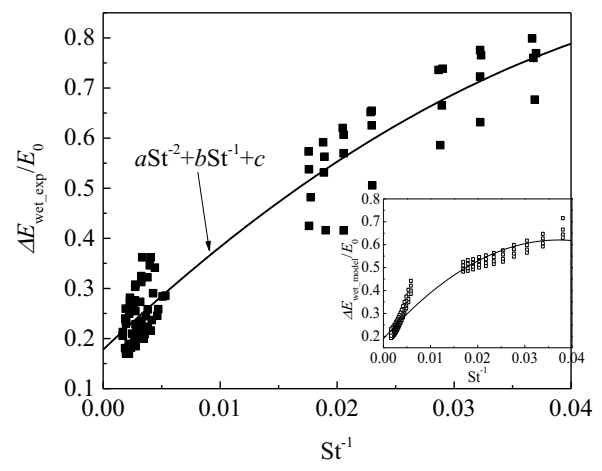

Fig. 5. The ratio of $\Delta E_{\text {wet }}$ to $E_{0}$ varies with $\mathrm{St}^{-1}$ by experiment in reference [10] (inset is the result predicted by our theoretical model).

\section{Conclusions}

In this paper, the forces that the particle contacts with a wet wall in both quasi-static and dynamic state are performed. First, capillary force is measured by AFM between a micron-sized particle and a glass plate. It is found out that the capillary force begins to grow slowly with $\mathrm{RH}$, and then increases rapidly. This result is explained by capillary condensation that liquid only exists in the concave of the surface roughness below a critical RH, but it fills up the gap beyond a critical value.

Second, the colliding process that a falling grain impacting onto a wet wall is analyzed. Compared with the methodology of energy budget referred in the previous literature, the force models presented here could not only calculate the first-contact energy loss, but also study the contact forces and particle velocities at any given time. The simulation results show that our model agrees well with the experimental data and are adaptive in both low and high viscosity fluid cases.

\section{Acknowledgements}

This work was funded by the China Postdoctoral Science Foundation and the Major State Basic Research Development Program of China (Grant No. 2016YFC0203705).

\section{References}

1. S. Herminghaus, Adv. Phys. 54, 221 (2005).

2. J. Delenne, V. Richefeu, F. Radjai, J. Fluid Mech. 762, R5 (2015).

3. B. Andreotti, Y. Forterre, O. Pouliquen, Granular media between fluid and solid (Cambridge University Press, New York, 2013).

4. O. Pitois, P. Moucheront, X. Chateau, J. Colloid Interface Sci. 231, 26 (2000).

5. O. Pitois, P. Moucheront, X. Chateau, Eur. Phys. J. B 23, 79 (2001).

6. J. Marshall, S. Li, Adhesive particle flow a discreteelement approach (Cambridge University Press, New York, 2014).

7. R. Jones, H. Pollock, J. Cleaver, C. Hodges, Langmuir 18, 8045 (2002).

8. P. Liu, R. Yang, A. Yu, Chem. Eng. Sci. 86, 99 (2013).

9. S. Chen, S. Li, M. Yang, Powder Technol. 274, 431 (2015)

10. F. Gollwitzer, I. Rehherg, C. Kruelle, K. Huang, Phys. Rev. E 86, 011303 (2012).

11. S. Antonyuk, S. Heinrich, N. Deen, H. Kuipers, Particuology 7, 245 (2009).

12. D. Lee, H. Kim, Langmuir 24, 142 (2008).

13. J. Simeonov, Acta. Mech. 227, 565 (2016).

14. E. Izard, T. Bonometti, L. Lacaze, J. Fluid Mech. 747, 422 (2014).

15. D. Vella, Langmuir 22, 2972 (2006).

16. A. Wang, Q. Song, Q. Yao, Atmos. Environ. 115, 1 (2015)

17. R. Zhu, S. Li, Q. Yao, Phys. Rev. E 87, 022206 (2013). 